Acta vet. scand. 1964, 5, 179-187.

Aus dem Veterinärmedizinischen Staatsinstitut, Stockholm, Schweden.

\title{
INTRANASALE VAKZINATION VON MEERSCHWEINCHEN MIT EINEM AVIRULENTEN STAMM DER PASTEURELLA PSEUDOTUBERCULOSIS
}

Von

\author{
E. Thal, E. Hanko und W. Knapp ${ }^{\star}$ )
}

Ausgehend von den früheren Beobachtungen, dass mit dem avirulenten Stamm 32IV von $P$. pseudotuberculosis nach subkutaner, intraperitonealer und peroraler Applikation eine solide Immunität gegen eine massive Infektion mit virulenten Stämmen von $\boldsymbol{P}$. pestis und $\boldsymbol{P}$. pseudotuberculosis erzielt wird (Thal, 1954, 1955, 1956, 1962), prüften wir die Frage inwieweit die intranasale Applikation des Stammes $32^{\mathrm{IV}}$ von einer Immunität gegen virulente $\boldsymbol{P}$. pseudotuberculosis-Keime an Meerschweinchen gefolgt ist. Anlass zu diesen Untersuchungen war ausserdem der Hinweis von $K$. F. Meyer (1961), zu experimentellen Untersuchungen beim Meerschweinchen über Lungenpest, dass eine Infektion des Respirationstractus gewöhnlich wesentlich schwerer verläuft als die kutane und subkutane. Sie sei daher besonders gut geeignet um den Wert von Impfstoffen und den Wert der erzielten Immunität zu prüfen. Gleichzeitig sollten die Versuche zeigen, ob und welche Unterschiede bei den Lungenveränderungen der infizierten Tiere einerseits und der vakzinierten und anschliessend infizierten Tiere andererseits nachgewiesen werden können. Schliesslich war die Dauer der Persistenz der intranasal applizierten Keime in der Lunge und ihre Ausbreitung auch in anderen Organen sowie das Auftreten humoraler Antikörper zu prüfen.

Herrn Professor emeritus Dr. K. F. Meyer, San Francisco, zu seinem 80ten Geburtstag in Verehrung gewidmet.

*) Professor Dr. med. W. Knapp, Hygienisch-Bakteriologisches Institut der Universität Bern, Schweiz. 


\section{UNTERSUCHUNGSTECHNIK}

\section{Impfungs- und Infektionsverfahren.}

Die für die Versuche verwendeten Meerschweinchen (Gewicht c:a $300 \mathrm{~g}$ ) wurden in 3 Gruppen eingeteilt:

Gruppe A: 7 Meerschweinchen intranasal geimpft und infiziert. Die Impfung erfolgte durch langsames Aufsetzen von je 8 Tropfen $(0.4 \mathrm{ccm})$ einer 48-stündigen Bouillonkultur von Stamm 32Iv (c:a $3 \cdot 10^{8} \mathrm{Keime} / \mathrm{ccm}$ ) auf die Nase mit Aether narkotisierter Meerschweinchen. Die Infektion der Tiere erfolgte nach 5 Wochen in derselben Weise mit dem virulenten Stamm 14I (c:a $\left.6 \cdot 10^{8} \mathrm{Keime} / \mathrm{ccm}\right)$. Alle Tiere wurden 4 Wochen nach der Infektion zum Nachweis makroskopischer und histologischer Organveränderungen und einer eventuellen Persistenz des Impf- und Infektionsstammes getötet.

Gruppe B: 6 Meerschweinchen geimpft, aber nicht infiziert (1. Kontrollgruppe).

Die Impfung erfolgte wie bei der Gruppe A. Zum Nachweis der Organveränderungen und einer eventuellen Persistenz des Impfstammes wurden 3 Tiere nach 10, 1 Tier nach 20 und 2 Tiere nach 30 Tagen getötet und pathologisch-anatomisch sowie bakteriologisch untersucht.

\section{Gruppe C: 6 Meerschweinchen infiziert (2. Kontrollgruppe).}

Die Infektion erfolgte wie bei der Gruppe A. Vier Tiere erlagen der Infektion nach 8, 10, 11 bzw. 13 Tagen, während das fünfte und sechste Tier am 30. Tag p.i. getötet wurden. Alle Tiere wurden ebenfalls pathologisch-anatomisch und bakteriologisch untersucht.

\section{Bakteriologisch-serologische Untersuchungen.}

Von allen Tieren wurden mindestens folgende Organe, auch wenn sie keine makroskopisch sichtbaren Veränderungen zeigten, bakteriologisch untersucht: Lungen, Lungen- und Mesenteriallymphknoten, Milz und Leber. Andere Organe wurden nur, wenn sie Veränderungen zeigten, auf Erreger untersucht. Der kulturelle Erregernachweis erfolgte durch Beimpfen von Bouillon, modifiziertem Drigalski- und Desoxycholatcitratagar. Verdächtige Kolonien wurden nur dann als P. pseudotuberculosis Typ I (Stamm 14) bzw. Typ IV (Stamm 32) diagnostiziert, wenn sie in den kulturell-biochemischen und serologischen Untersuchungen wie auch im Phagentest mit dem PSTPhagen, die diagnostischen Voraussetzungen erfüllten.

Bei allen Tieren der A- und B-Gruppe und den beiden am 30. Tag getöteten Tieren der C-Gruppe wurde versucht mittels der Agglutinationsmethode Antikörper gegen den Impfstamm (32IV) bzw. den Infektionsstamm (14I) nachzuweisen. 


\section{Pathologisch-anatomische Untersuchungen.}

Sämtliche Tiere wurden nach der Sektion makroskopisch und histologisch untersucht. Die histologischen Präparate wurden in üblicher Weise mit Formalin fixiert und die Schnitte nach van Gieson und mit Hämatoxylin-Eosin gefärbt.

\section{ERGEBNIS DER UNTERSUCHUNGEN}

Bakteriologisch-serologische Befunde.

Ein Nachweis des Impfstammes war bei keinem Tier der A-Gruppe und des Infektionsstammes nur bei einem Tier aus einem Lungenlymphknoten mit Hilfe der Bouillonanreicherung, aber nicht in der Direktaussaat auf den beiden festen Medien möglich. Bei den 6 nur vakzinierten Tieren der B-Gruppe gelang in zwei Fällen der Nachweis des Impfstammes. Stamm 321v wurde bei dem am zehnten Tag getöteten Tier aus Lunge und Milz und bei dem zweiten erst am 30. Tag getöteten Tier nur aus einem Lungenlymphknoten gezüchtet. Bei den spontan gestorbenen und den zwei getöteten Tieren der Gruppe $\mathrm{C}$ konnte der Infektionsstamm aus den meisten Organen isoliert werden.

Ein deutlicher Agglutinintiter gegen den Infektions- bzw. Impfstamm war in keiner Serumprobe nachweisbar, auch nicht im Serum der beiden am 30. Tag getöteten Tiere der Gruppe C, d. h. sämtliche Agglutinationswerte hielten sich bei oder unter $1: 10$.

Pathologisch-anatomische Befunde.

Die Tiere der Gruppe A, die bei der Sektion ausser kleinen artifiziell bedingten Blutungsherden (Tötung) keine makroskopisch sichtbaren Veränderungen zeigten (Abb. 1), boten auch bei der histologischen Untersuchung ein weitgehend einheitliches Bild, sodass auf die Wiedergabe der einzelnen durch Präparate und protokollarisch festgestellten Befunde verzichtet werden kann. Wiederholt wurde folgender Befund erhoben:

Makroskopisch: negativ

Mikroskopische Untersuchungen:

Lungen: Geringe herdförmige Hypertrophie und Proliferation des Alveolarepithels. Unterschiedlich stark ausgeprägte lymphohistiozytäre Infiltrate peribronchiell und perivaskulär sowie in den Alveolarsepten (Abb. 2). 


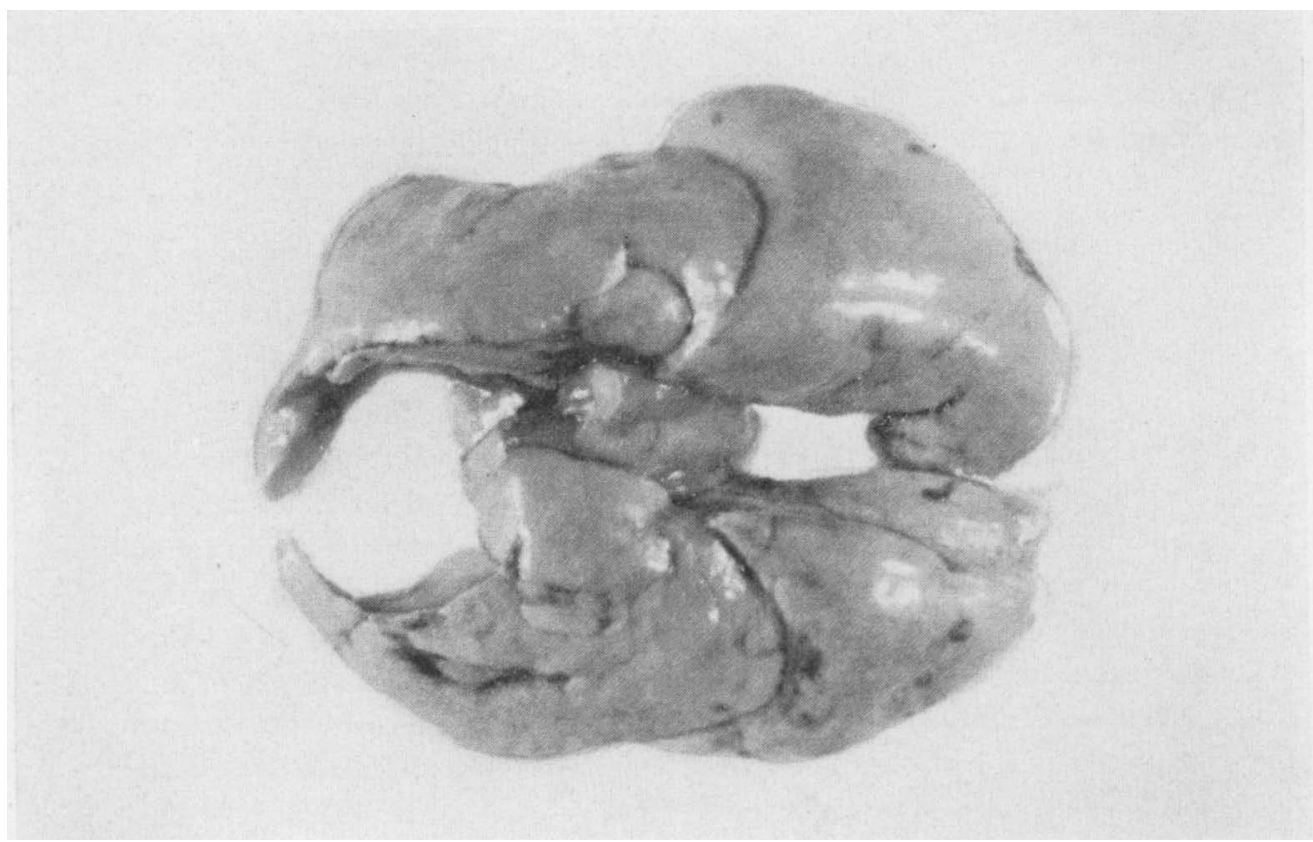

A b bildung 1: Lungen von Meerschweinchen der Gruppe A (vakziniert $u$. infiziert) normal mit kleinen Blutungsherden.

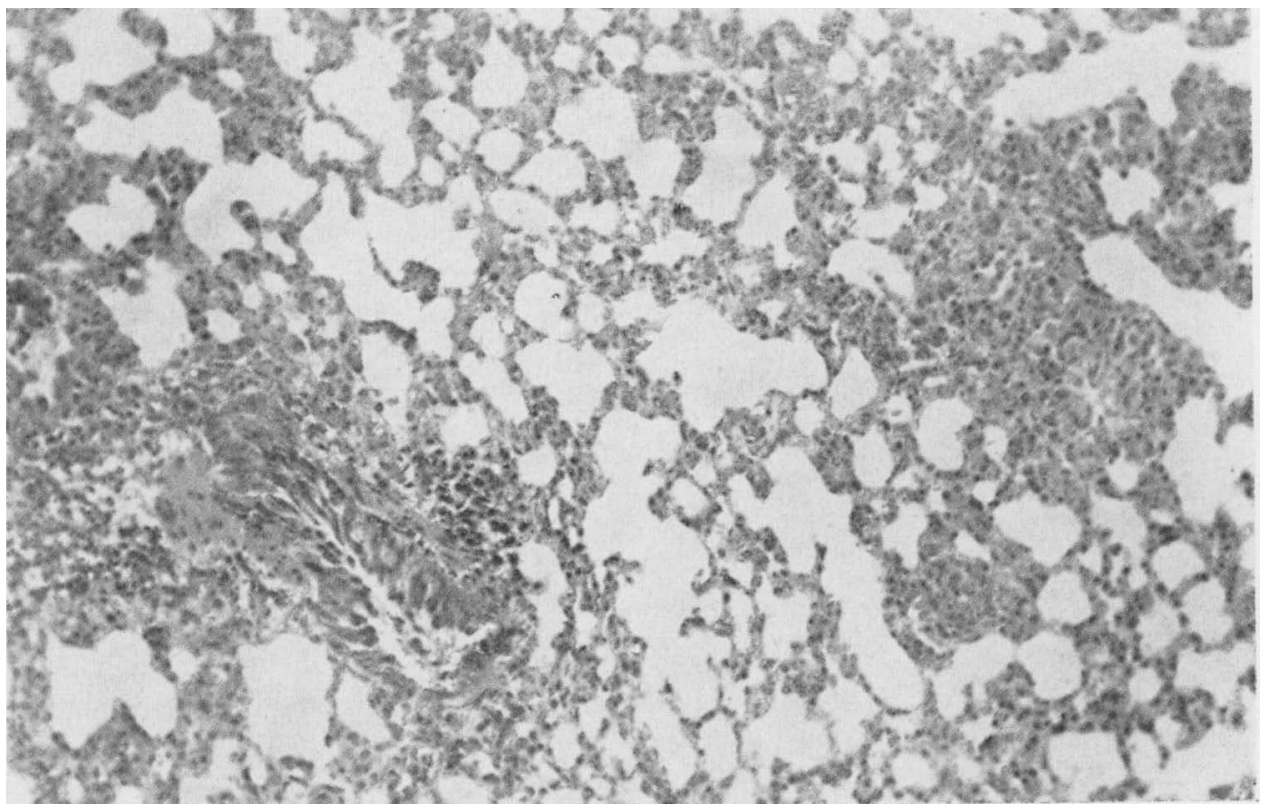

A b bildung 2: dto., mikroskopisches Bild, Vergr. $\times 150$, mit Hämatoxylin-Eosin gefärbt. Vereinzelte peribronchielle lymphohistiozytäre Infiltrate und leichte Alveolarepithelproliferation. 


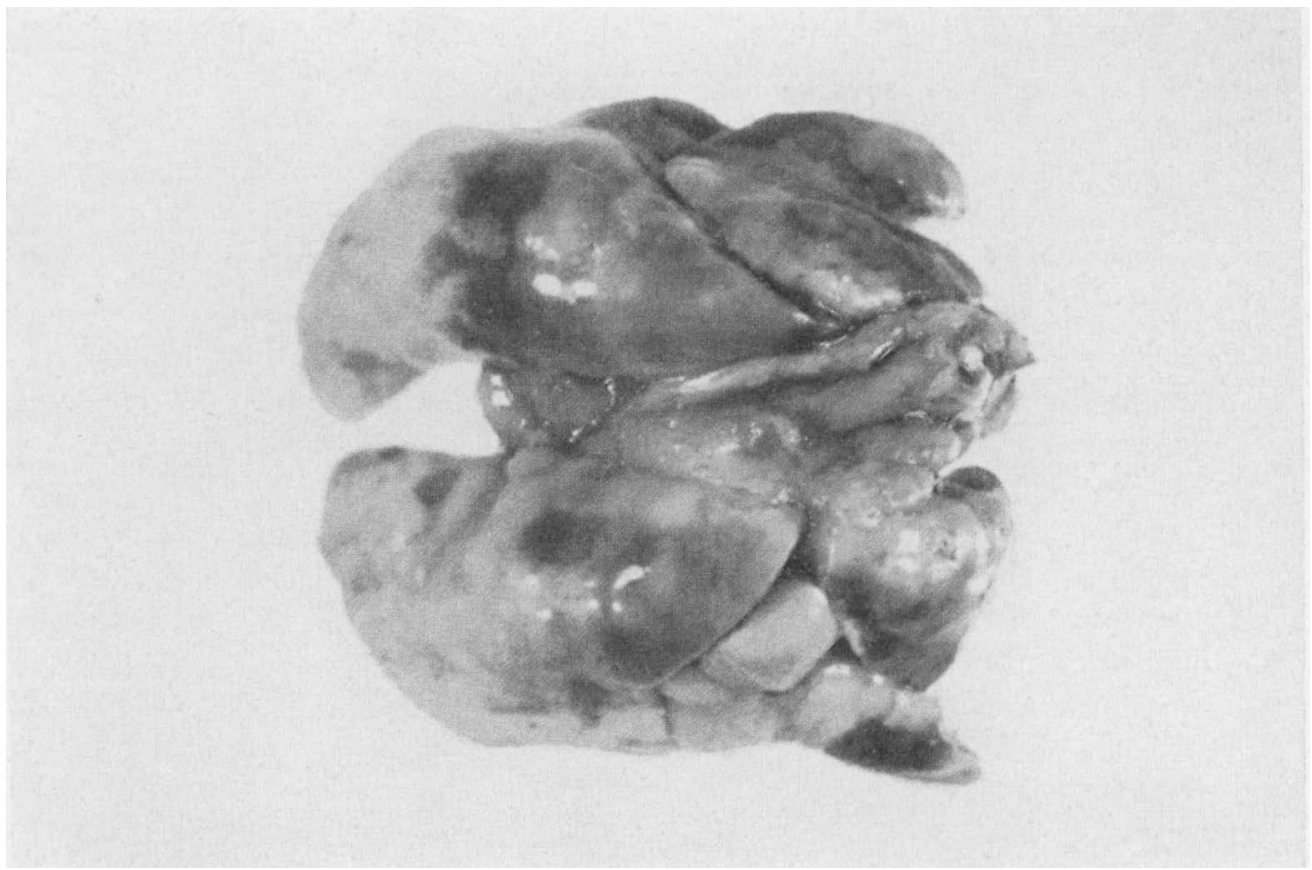

A b bild ung 3: Lungen von Meerschweinchen der Gruppe C (nur infiziert) ausgedehnte, abszedierende pneumonische Herde in den oberen Teilen der Lungen.

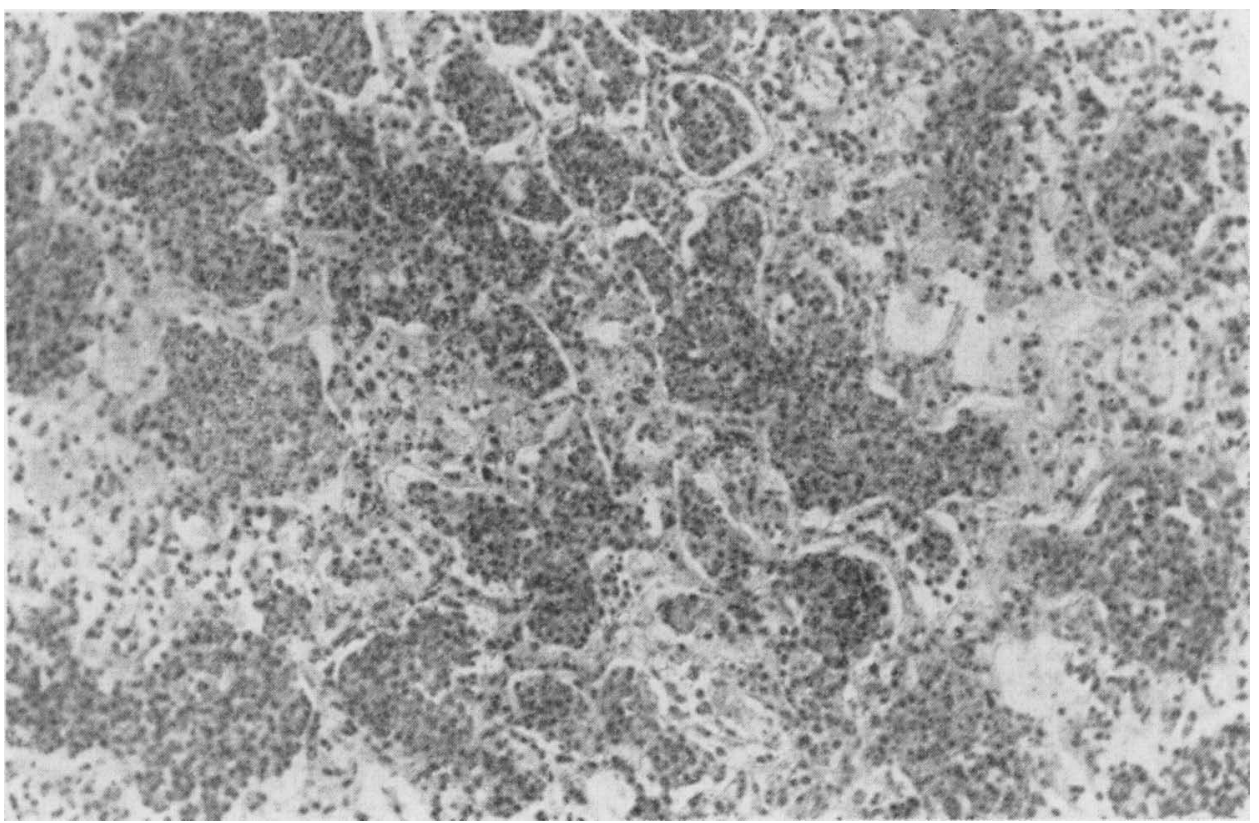

A b bildung 4: dto., mikroskopisches Bild, Vergr. $\times 150$, mit Hämatoxylin-Eosin gefärbt, ausgedehnte purulent-abszedierende Pneumonie. 
Milz: Schwache follikuläre Hyperplasie; in der roten Pulpa vereinzelte reife und unreife Plasmazellen.

Leber und extrapulmonale Lymphknoten: ohne Veränderungen.

Dieselben makroskopischen und histologischen Befunde wurden bei den Tieren der Gruppe B (1. Kontrollgruppe) gefunden. Dabei waren die histologischen Befunde - von graduellen Unterschieden abgesehen - weitgehend dieselben, ob die Tiere 10, 20 oder 30 Tage nach Impfung mit dem avirulenten Stamm getötet und untersucht wurden. Eine Wiedergabe der Befunde erübrigt sich.

Bei den Tieren der Gruppe C (2. Kontrollgruppe - nur infizierte Tiere) wurden dagegen makroskopisch und mikroskopisch ausgedehnte Lungenveränderungen nachgewiesen. Die bei den einzelnen spontan gestorbenen oder getöteten Tieren erhobenen Befunde variierten besonders in ihrem Ausmass und weniger in ihrer Art, sodass auch über sie zusammenfassend berichtet werden kann.

Makroskopisch: Herdpneumonien unterschiedlicher Ausdehnung mit zum Teil grauen abszessartigen Herden (Abb. 3). Unterschiedlich stark vergrösserte Bronchiallymphknoten von vermehrter Konsistenz. Bei 2 Tieren die Milz deutlich vergrössert. Uebrige Organe makroskopisch unverändert.

Mikroskopische Untersuchung:

Lungen: Unterschiedlich starke akut-subakute oder subakutchronische abszedierende Pneumonie; umgebendes Lungenparenchym in der Regel hyperämisch und ödematös. Wechselnd starke retikulozytäre-lymphozytäre Hyperplasie der Bronchiallymphknoten mit zum Teil zahlreichen nekrotischen Herden. Mässige Proliferation des Alveolar- und Bronchialepithels (Abb. 4).

Milz: Bei allen Tieren unterschiedlich starke Hyperplasie der Follikel mit Nachweis retikulumzelliger Infiltrate.

Leber und extrapulmonale Lymphknoten: ohne Veränderungen.

\section{BESPRECHUNG DER ERGEBNISSE}

Unsere Untersuchungsergebnisse ermöglichen folgende Feststellungen:

a) Wie die orale, führt auch die intranasale Impfung mit dem avirulenten oder sehr stark virulenz-abgeschwächten Stamm 32rv von $P$. pseudotuberculosis zu einer sicheren Immunität gegen virulente heterologe $P$. pseudotuberculosis-Stämme. 
b) Die Sektionsbefunde und histologischen Untersuchungen der Tiere aller 3 Gruppen liessen für die infizierten Kontrolltiere u. a. eine akut-subakute oder subakut-chronische abszedierende Pneumonie nachweisen, während diese bei den geimpften bzw. geimpften und anschliessend infizierten Tieren nicht beobachtet werden konnte. Diese Tiere zeigten nur geringgradige Lungenveränderungen wie sie zum Teil in der Meerschweinchenlunge schon auf geringfügige Reize nicht nur bakterieller, sondern auch mechanischer Art, d. h. nach den verschiedensten Eingriffen, beobachtet werden können (Cohrs, Jaffé u. Meessen, 1958). Die von uns nach der intranasalen Infektion beobachteten Befunde entsprechen weitgehend den von Masshoff (1962) nach intratrachealer Infektion tracheotomierter Meerschweinchen beschriebenen Lungenveränderungen. Während bei unseren Tieren das Bild der abszedierenden Pneumonie im Vordergrund stand, sah Masshoff vor allem grossflächige Nekrosen. Diese unterschiedlichen Befunde sind wahrscheinlich als Folgen verschieden grosser Infektionsdosen und gradueller Unterschiede in der Virulenz der für die Infektion verwendeten Stämme zu deuten.

c) Die bakteriologischen Untersuchungen liessen einerseits eine Generalisation des Impfstammes durch seine Züchtung aus der Milz 10 Tage nach der Impfung und andererseits seine Persistenz in Lungenlymphknoten bis zum Abschluss der Beobachtungszeit von 30 Tagen nachweisen. Auch eine Persistenz des Infektionsstammes in den Lungenlymphknoten der schutzgeimpften Tiere ist möglich. Seine Generalisation konnte bei diesen Tieren nicht nachgewiesen werden. Die Beobachtung kann wohl damit erklärt werden, dass die Keime entweder nach der Generalisation rasch zugrunde gegangen und deshalb am 10. Tag nach der Infektion nicht extrapulmonal nachweisbar waren oder die Generalisation blieb als Folge der guten Immunität der intranasal immunisierten und infizierten Meerschweinchen aus. Eine endgültige Beantwortung dieser Frage setzt weitere bakteriolodische Untersuchungen an geimpften und infizierten Tieren bereits in den ersten Tagen nach der Infektion voraus.

d) Das Fehlen von Agglutininen bzw. der Nachweis eines nur sehr niederen Agglutinintiters (1:10) wird mit allem Vorbehalt dahingehend gedeutet, dass wie für die Pest bekannt (Meyer 1950, Girard 1955) auch bei der Pseudotuberkulose die Immunität zellulärer und nicht humoraler Natur ist. Für diese An- 
nahme sprechen auch die Untersuchungen von Rutqvist, Thal u. Åberg (1956), denen es nicht gelang mit hochtitrigen Seren eine passive Immunisierung durchzuführen.

e) Unsere bakteriologischen Untersuchungen zeigten erneut die Ueberlegenheit der flüssigen Nährböden, als Anreicherungsmedium, bei der Isolierung weniger, möglicherweise schon geschädigter Pasteurella-Keime aus Organmaterial und damit die Notwendigkeit die Kulturen gleichzeitig in flüssigen und auf festen Medien anzulegen und über mehrere Tage zu bebrüten (Knapp 1960).

\section{LITERATURVERZEICHNIS}

Cohrs, P., R. Jaffé \& H. Meessen: Pathologie der Laboratoriumstiere, Springer-Verlag, 1958, 35-36.

Girard, G.: Plague, Ann. Rev. Microbiol., 1955, 9, 253-276.

Knapp, W.: Die Laboratoriumsdiagnose von Infektionen mit Pasteurella pseudotuberculosis. Das ärztliche Laboratorium, 1960, 6, 197-206.

Masshoff, W.: Die Pseudotuberkulose des Menschen, Dtsch. med. Wschr. 1962, 87, 915-920.

Meyer, K. F.: Immunity in Plague; critical consideration of some recent studies, J. Immunol., 1950, 64, 139-163.

Meyer, K. F.: Pneumonic Plague, Bact. Rev., 1961, 25, 249-261.

Rutqvist, L., E. Thal \& E. A Bberg: Changes in the serum proteins of guinea pigs immunized with Pasteurella pseudotuberculosis. Acta Path. Microbiol. Scan., 1956, 39, 94-102.

Thal, E.: Studies on Pasteurella pseudotuberculosis with special reference to its immunological behaviour. (Untersuchungen über Pasteurella pseudotuberculosis unter Berücksichtigung ihres immunologischen Verhalten) Nord. Vet.-Med., 1954, 6, 829-832.

Thal, E.: Immunisierung gegen Pasteurella pestis mit einem avirulenten Stamm der Pasteurella pseudotuberculosis. Nord. Vet.-Med., $1955,7,151-153$.

Thal, E.: Relations immunologiques entre Pasteurella pestis et Pasteurella pseudotuberculosis. Ann. Inst. Pasteur, 1956, 91, 68-74.

Thal, E.: Oral immunization of guinea pigs with avirulent Pasteurella pseudotuberculosis, Nature, 1962, 194, 490—491.

\section{ZUSAMMENFASSUNG}

Es wird über die erfolgreiche intranasale Impfung von Meerschweinchen mit dem avirulenten Pasteurella pseudotuberculosisStamm 32IV berichtet. Die bakteriologischen und pathologisch-anatomischen Befunde bei den in drei Gruppen eingeteilten - vakzinierten, vakzinierten und infizierten und nur infizierten - Meerschweinchen werden beschrieben. 


\section{SUMMARY}

Intranasal vaccination of guinea pigs with an avirulent strain of Pasteurella pseudotuberculosis.

Successfull vaccination was achieved by intranasal instillation of guinea pigs with avirulent strain $32^{\mathrm{IV}}$ of Pasteurella pseudotuberculosis. The bacteriological findings and the pathological changes in the 3 groups of guinea pigs - vaccinated, vaccinated and infected and only infected - are described.

\section{SAMMANFATTNING}

Intranasal vaccination av marsvin med en avirulent Pasteurella pseudotuberculosis-stam.

Framgångsrik vaccination kunde åstadkommas genom intranasal ympning på marsvin med den avirulenta Pasteurella pseudotuberculosis-stammen 32IV. De bakteriologiska fynden och de patologisk-anatomiska förändringarna hos de i 3 grupper indelade marsvinen vaccinerade, vaccinerade och infekterade samt endast infekterade beskrivs.

(Received September 4. 1963). 